\title{
Estudo retrospectivo dos níveis de ácido hipúrico urinário em exames de toxicologia ocupacional
}

\author{
A retrospective study analysis of urinary hippuric acid levels \\ in occupational toxicology exams
}

Kelly Cristina Gonzalez ${ }^{1}$

Fernando Rodrigues Sagebin ${ }^{2}$

Paola Garcia O liveira ${ }^{1}$

Luiz Glock ${ }^{1}$

FlaviaValladão Thiesen ${ }^{2}$

${ }^{1}$ FaculdadedeFarmácia, Pontifícia Universidade Católica do Rio Grandedo Sul.Av. I piranga 6681,

Prédio 12A, sala 202,

Partenon. 90619-900

Porto Alegre RS.

fvthiesen@pucrs.br

${ }^{2}$ Instituto de Toxicologia,

Pontifícia Universidade

Católica do Rio Grande do Sul.
Abstract $\mathrm{Hippuric}$ acid is the primary metabolite of toluene, a solvent widely used in industrial processes with considerable toxic effects, a fact which justifies regularly monitoring individuals with occupational exposure to this solvent. This work aims at evaluating urinary hippuric acid levels found in workers subject to biological monitoring. A retrospectivestudy was carried out with data referring from 2002 to 2005, in which exams results and employment status wereanalyzed ( $p e-$ riodic, post-employment, and pre-employment exams). Results indicate a significant reduction in hippuric acid levels for 2005. Periodic exams presented higher results than pre-employment and post-employment exams. No significant difference was found in individuals grouped according to their status in each of the established intervals, their reference numbers, and maximum biological levels allowed. Hippuric acid levels detected indicate low risk of toluene exposure for the population under evaluation, probably due to a growing concern with the deployment of measures regarding occupational hygiene. Key words Toluene, Hippuric acid, O ccupational exams, Biological monitoring
Resumo 0 ácido hipúrico éo principal metabóli to do tolueno, solventeamplamente utilizado em processos industriais e com importantes efeitos tóxicos, fato que justifica a preocupação em monitorar regularmente sujeitos com risco de exposição ocupacional a este solvente. 0 objetivo deste trabalho foi avaliar os níveis de ácido hipúrico encontrados em trabalhadores submetidos à monitorização biológica. Foi realizado um estudo retrospectivo com dados dos anos de 2002 a 2005, no qual foram analisados os resultados ea situação do empregado na oportunidade do exame (periódico, demissional e admissional). Os resultados indicam uma redução significativa nos níveis de ácido hipúrico em 2005. Exames periódicos obtiveram resultados superiores aos exames admissionais e demissionais, enão foi verificada diferença significativa nas proporções dos sujeitos agrupados de acordo com a situação funcional em cada um dos intervalos estabelecidos segundo 0 valor de referência e 0 índice biológico máximo permitido. Os níveis de ácido hipúrico detectados indicam um baixo de risco de exposição ao tolue no na população avaliada, provavel mente em de corrência da preocupação crescente com a implantação de medidas de higiene ocupacional. Palavras-chave Tolueno, Ácido hipúrico, Exames ocupacionais, M onitorização biológica 
Introdução

0 tolueno éum hidrocarboneto aromático volátil eincolor com amplo uso industrial, naturalmente presenteno petróleo. É um dos solventes produzidos em maior quantidade ecerca de $92 \%$ do tolueno obtido são utilizados na produção de gasolina. 0 restante, purificado como tolueno comercial, é empregado na produção de produtos quími$\cos ^{1-3}$. Muitas tintas, colas, polidores, diluentes, desengordurantes e removedores contêm estesolvente como seu principal constituinte ${ }^{4-6}$.

É um solvente que atua sobre o sistema nervoso central (SNC) em exposições agudas e crônicas. Apresenta ação irritante sobre peleemucosas e seus efeitos agu dos são semelhantes aos de correntes da intoxicação etanólica: efeitos estimulantes seguidos de depressão do SNC. Em exposições crônicas, pode provocar hepatotoxicidade, nefrotoxicidade e perda auditiva?. Estudos sobre a exposição crônica ao tolueno a baixas concentrações demonstram mudanças neurocomportamentais e exposições a altas concentrações podem ocasionar não apenas déficits de atenção e concentração, mas também motores ${ }^{6}$. 0 controle e prevenção da exposição a este solventeé realizado através de medidas de controle ambiental, uso de roupas impermeáveis, luvas, óculos desegurança e máscaras com filtro químico ${ }^{3}$. Adicionalmente, tendo em vista seus efeitos tóxicos, todos os trabalhadores com risco de exposição ao tolueno devem realizar monitorização biológica por meio de exames toxicológicos para avaliar seu grau de exposição a este solvente.

A Norma Regulamentadora no 7 (NR-7), da Secretaria de Segurança e M edicina do Trabal ho do M inistério do Trabalho ${ }^{8}$, indica o ácido hipúrico $(\mathrm{AH})$ urinário como indicador de exposição ocupacional ao tolueno. $\mathrm{O}$ AH éo metabólito do tolueno encontrado em maior concentração na urina e seus níveis estão diretamente relacionados aos níveis de tolueno no ambiente, sendo considerado adequado como biomarcador de exposição a este solvente ${ }^{9,10}$. De acordo com a NR-7, o valor de referência (VR) para o AH é 1,5 grama de ácido hipúrico por grama de creatinina e seu índice biológico máximo permitido (IBM P) é 2,5 gramas de ácido hipúrico por grama de creatinina ${ }^{8}$.

Considerando os efeitos tóxicos do tolueno e o grande número de trabal hadores com risco de exposição a este solvente, foi realizado um estudo retrospectivo com o objetivo de avaliar os níveis de $\mathrm{AH}$ detectados nos trabalhadores submetidos à monitorização biológica.
Metodologia

Foi realizado um estudo retrospectivo descritivo, utilizando a base de dados do Instituto de Toxicologia da Pontifícia Universidade Católica do Rio Grande do Sul. Foram utilizados os dados das dosagens de $\mathrm{AH}$ urinário no período de 2002 a 2005. 0 ácido hipúrico foi analisado por cromatografia gasosa com detector de ionização em chama ecorrigido por creatininúria. A sensibilidade do método empregado é de $0,1 \mathrm{~g} / \mathrm{L}, 0$ limite de detecção é $0,05 \mathrm{~g} / \mathrm{L}$ e o método élinear no intervalo de 0,1 a $3,0 \mathrm{~g} / \mathrm{L}$. A totalidade das análises realizadas no período citado $(\mathrm{N}=2279)$ foi submetida à análise descritiva com o objetivo de verificar o perfil dos níveis de ácido hipúrico nostrabalhadores queutilizam o tolueno em suas atividades laborais.

Foram calculados as médias, os desvios padrão e os erros padrão para as variáveis quantitativas (níveis de AH urinário), enquanto as variáveis categóricas (ano da coleta, situação funcional em queforam realizados os testes - admissional, demissional ou periódico, concentração de AH na urina - acima do VR, entre VR e IBM $P$, acima do (BM $P$ ) foram descritas em função de suas percentagens. A associação entre as variáveis qualitativas foi calculada empregando o teste do qui-quadrado. A associação entre as variáveis quantitativas, agrupadas segundo as categorias ano de coleta e situação funcional em que foram realizados os testes, foram comparadas através da análise de variância (Anova) com pósteste de Duncan. Em todos os casos, foram consideradas significativas as associações quando a probabilidade $P$ dos eventos assumiu valores iguais ou inferiores a 0,05 . O s cálculos estatísticos foram executados em planilhas do SPSS, versão 11.5.

\section{Análiseediscussão dos resultados}

A Figura 1 apresenta resultados da média e intervalo de confiança (95\%) dos valores de ácido hipúrico para os diferentes anos da pesquisa. Submetida à análise de variância, observou-se diferença significativa entre os sujeitos da amostra agrupados segundo 0 ano de coleta $(P=0,0001)$. Verificou-se, através do teste de pós-testedeDuncan, que 0 ano de 2005 aparece com um valor significativamente menor que os demais, entreos quais não se verificou diferença significativa. É possível observar claramentequeo espaço de dispersão dos dados de 2005 não se confunde com 
nenhum ano anterior. Pode-se atribuir a redução significativa do ácido hipúrico nos sujeitos com dados coletados em 2005 a um processo de amadurecimento das campanhas dehigieneocupacional quevêm sendo implementadas exaustivamente ao longo deste período por núcleos de segurança do trabalho das empresas. Outra hipótese é a redução do uso do tolueno, de modo semelhante ao que está ocorrendo em alguns países ${ }^{11}$.

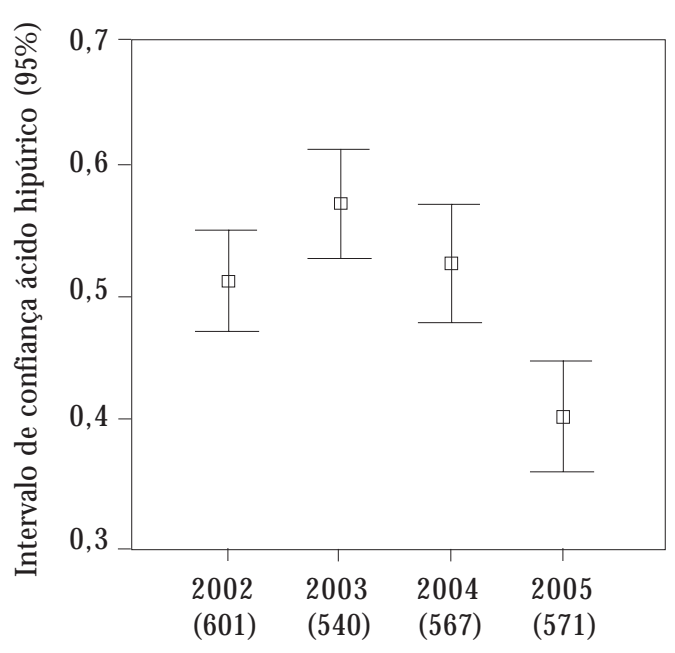

Ano e no de exames

Figura 1. M édia e dispersão dos valores de ácido hipúrico para os diferentes anos da pesquisa.
Os dados de valores da média, desvio padrão e erro padrão do ácido hipúrico urinário agrupados segundo situação do empregado na oportunidade do exame (periódico, demissional e admissional) são apresentados na Tabela 1. Após análise de variância, foi observada diferença significativa $(P=0,001)$ entre os grupos, destacando-se os exames periódicos com os valores significativamente mais elevados quando observado o resultado do teste de post hoc de Duncan. Este resultado é explicável pelo fato dos trabaIhadores estarem em plena atividade funcional quando são realizados os exames periódicos. Não houve diferença significativa entre os grupos dos exames admissionais e demissionais. 0 exame demissional, se realizado logo após o desligamento, deve detectar exposição a níveis mais el evados de tolueno. No entanto, devido à curta meia-vida do ácido hipúrico (meia-vida de excreção deduas a quatro horas) $)^{9,12}$, nota-se que 0 intervalo entre a requisição do examee a coleta da amostra pode influenciar neste resultado, levando à normalização dos níveis de $\mathrm{AH}$.

Os dados dos sujeitos agrupados segundo as variáveis qual itativas situação funcional econcentração deácido hipúrico na urina foram categorizados em 1, 2 e 3, segundo os critérios da NR-7. Desta forma, na categoria 1 estão as dosagens de $\mathrm{AH}$ abaixo de $1,5 \mathrm{~g} / \mathrm{g}$ decreatinina, na categoria 2 , aquelas entre 1,5 e2,5 g/g de creatinina e, na categoria 3, foram incluídos todos os resultados maiores que $2,5 \mathrm{~g} / \mathrm{g}$ de creatinina (Tabela 2).

Tabela 1. Níveis de ácido hipúrico dos participantes de acordo com a situação do funcionário no momento de realização do exame ( $\mathrm{N}=2279)$.

\begin{tabular}{lcccc}
\hline Situação funcional & Número de exames & Média ( $/$ / g de creatinina) & D esvio padrão & Erro padrão \\
\hline Periódico & 1165 & 0,540 & 0,5098 & 0,0149 \\
Demissional & 311 & 0,458 & 0,4387 & 0,0249 \\
Admissional & 803 & 0,459 & 0,5673 & 0,0200 \\
Total & 2279 & 0,500 & 0,5234 & 0,110
\end{tabular}

Tabela 2. Situação funcional dos participantes e concentração de ácido hipúrico categorizada de acordo com a NR-7.

\begin{tabular}{lrrrr}
\hline & Periódico & Demissional & Admissional & Total \\
\hline Ácido hipúrico categorizado & & & & \\
$1(<1,5$ g/g de creat) & 1100 & 299 & 756 & 2155 \\
$2(1,5$ a 2,5 g/g de creat) & 57 & 11 & 36 & 104 \\
$3(>2,5$ g/g de creat) & 8 & 1 & 11 & 20 \\
Total & 1165 & 311 & 803 & 2279 \\
\hline
\end{tabular}


A pós aplicar o teste de qui-quadrado, não foi verificada diferença significativa nas proporções dos sujeitos agrupados segundo a situação funcional em cada um dos valores da norma.

A maior utilização do ácido hipúrico como indicador biológico ocorrena avaliação da exposição de grupos de trabalhadores. Sua aplicação como indicador de exposição individual ao tolueno é limitada a exposições elevadas, já que seus níveis endógenos são altos e existe grande variação interindividual ${ }^{9}$. É importante salientar que este marcador biológico pode sofrer alterações; portanto, deve-seconhecer seus interferentes. No caso do tolueno, o AH é também um metabólito natural do organismo humano, podendo ser originado de dietas ricas em alimentos que contenham ácido benzóico e/ou seus precursores. Assim, a ingestão degrandes quantidades de refrige rantes ou enlatados conservados com ácido benzóico, café, chá da Índia, bebidas à base de matee ameixas, entre outros alimentos, pode elevar os níveis deácido hipúrico naurina9. O utra causa de aumento de ácido hipúrico urinário é o tabagismo, pois estima-se que um fumante inale de 80 a $160 \mu \mathrm{g}$ de tolueno por cigarro consumido ${ }^{13}$.

\section{Conclusões}

Apesar dos níveis de AH urinário serem significativamente maiores nos exames periódicos, os níveis encontrados neste estudo, em sua grande maioria, denotam que medidas de higiene ocupacional foram adequadamente empregadas. Estas medidas previnem a exposição dos trabaIhadores ao tolueno, evitando danos a sua saúde. Além disto, o fato dos valores encontrados serem menores no último ano avaliado indica uma preocupação crescentecom medidas de proteção no caso da população investigada. 


\section{Colaboradores}

KC Gonzalez participou da concepção, delineamento e redação do artigo; L Glock participou do delineamento e interpretação dos dados; PG de Oliveira participou da concepção e delineamento; FR Sagebin participou da concepção, delineamento, análise e interpretação dos dados e redação do artigo e FV Thiesen participou da concepção, delineamento, revisão crítica e aprovação da versão a ser publicada.

\section{Referências}

1. Eisenberg DP. Neurotoxicity and mechanism of toluene abuse. Einstein Quar J Biol M ed 2003;19:150-159.

2. Roberts LG, Bevans AC, Schreiner CA. Developmental and reproductive toxicity evaluation of toluene vapor in the rat. I Reproductive toxicity. Reprod Toxicol 2003;17:649-658.

3. Michel OR. Toxicologia ocupacional. Rio de Janeiro: Revinter; 2000.

4. El-Haj BM. A GC-M S method for the detection of toluene and ethylbenzene in volatile substance abuse. J Anal Toxicol 2000;24:390-394.

5. M athias R. Chronic solvent abusers have more brain abnormalities and cognitive impairments than cocaine abusers. NIDA N otes 2002; 17(4):6-12.

6. Kang SK, Rohlman DS, Lee MY, Lee HS, Chung SY, Anger WK. Neurobehavioral performance in workers exposed to toluene. Environ Toxicol Pharmacol 2005; 19(3):645-650.

7. Oga S. Fundamentos de toxicologia. $2^{\underline{a}}$ ed. São PauIo: Atheneu; 2003.

8. Brasil. Norma Regulamentadora $n^{\circ} 7$ (NR-7). Portaria no 24 de 29 de dezembro de 1994. Estabelece a obrigatoriedade de elaboração e implementação, por parte de todos os empregadores e instituições que admitam trabalhadores como empregados, do Programa de Controle M édico de Saúde Ocupacional - PCMSO, com o objetivo de promoção e preservação da saúde do conjunto dos seus trabalhadores. Diário Oficial da União 1994; 29 dez.

9. Fonseca EP, Johann I, Alegretti AP, Thiesen FV. Interferência do consumo de chimarrão nos níveis de ácido hipúrico urinário. Revista Brasileira de Análises Clínicas 2006; 38(3):163-165.

10. Fujii T, Kawabe S, Horike T, Taguchi T, Ogata M. Simultaneous determination of the urinary metabolites of toluene, xylene and styrene using highperformance capillary electrophoresis comparison with high-performance liquid chromatography. J Chromatogr B Biomed Sci Appl. 1999; 730:41-47.

11. Moriguchi J, Samoto H, Fukui Y, Ukai H, Okamoto S, Takada S, Ohashi F, Ezakia T, Ikeda M. Organic solvents used in enterprises of various sizes. Int Congr Ser 2006; 1294:218-221.

12. Brugnone F, Gobbi M, Ayyad K, Giuliari C, CerpelIoni $\mathrm{M}$, Perbellini L. Blood toluene as a biological index of environmental toluene exposure in the "normal" population and in occupationally exposed workers immediately after exposure and 16 hours later. Int Arch Occup Environ Health 1995; 66:421425.

13. Wang G, Maranelli G, Perbellini L, Guglielmi G, Brugnone $F$. Reference values for blood toluene in the occupationally nonexposed general population. Int Arch Occup Environ Health 1993; 65:201-203.

Artigo apresentado em 24/08/2007

Aprovado em 27/09/2007

Versão final apresentada em 23/10/2007 